\title{
Structural analysis of the $A \beta(11-42)$ amyloid fibril based on hydrophobicity distribution
}

\author{
Irena Roterman ${ }^{1} \cdot$ Dawid Dułak $^{2} \cdot$ Małgorzata Gadzała $^{3,4} \cdot$ Mateusz Banach $^{1} \cdot$ Leszek Konieczny $^{5}$
}

Received: 6 August 2018 / Accepted: 31 May 2019 / Published online: 10 July 2019

(c) The Author(s) 2019

\begin{abstract}
The structure of the $A \beta(11-42)$ amyloid available in PDB makes possible the molecular analysis of the specificity of amyloid formation. This molecule (PDB ID 2MVX) is the object of analysis. This work presents the outcome of in silico experiments involving various alternative conformations of the $\mathrm{A} \beta(11-42)$ sequence, providing clues as to the amylodogenecity of its constituent fragments. The reference structure (PDB) has been compared with folds generated using I-Tasser and Robetta-the strongest contenders in the CASP challenge. Additionally, a polypeptide which matches the A $\beta(11-42)$ sequence has been subjected to folding simulations based on the fuzzy oil drop model, which favors the production of a monocentric hydrophobic core. Computer simulations yielded 15 distinct structural forma (five per software package), which, when compared to the experimentally determined structure, allow us to study the role of structural elements which cause an otherwise globular protein to transform into an amyloid. The unusual positions of hydrophilic residues disrupting the expected hydrophobic core and propagating linearly along the long axis of fibril is recognized as the seed for amyloidogenic transformation in this polypeptide. This paper discusses the structure of the A $\beta(11-42)$ amyloid fibril, listed in PDB under ID $2 \mathrm{MXU}$ (fragment od $\mathrm{A} \beta(1-42)$ amyloid).
\end{abstract}

Keywords Amyloidosis · Fibrillation $\cdot A \beta(11-42) \cdot A \beta(1-42)$

Irena Roterman

myroterm@cyf-kr.edu.pl

Dawid Dułak

dawid.dulak@gmail.com

Małgorzata Gadzała

m.k.gadzala@gmail.com

Mateusz Banach

mateusz.banach@uj.edu.pl

Leszek Konieczny

mbkoniec@cyf-kr.edu.pl

1 Department of Bioinformatics and Telemedicine, Jagiellonian University - Medical College, Łazarza 16, 31-530 Kraków, Poland

2 ABB Business Services Sp. z o.o., Żegańska 1, 04-713 Warsaw, Poland

3 ACK - Cyfronet AGH, Nawojki 11, 30-150 Kraków, Poland

4 Present Address: Schibsted Tech Polska Sp. z o. o., Armii Krajowej 28, 30-150 Kraków, Poland

5 Chair of Medical Biochemistry, Jagiellonian University Medical College, Kopernika 7, 31-034 Kraków, Poland

\section{Introduction}

The volume of published papers which focus on amyloids is rapidly growing. Literature reviews [1] provide an up-to-date overview of current trends in protein misfolding research. This broad field encompasses various specific issues, such as the genetic underpinnings of amyloidogenesis and molecular studies [2], including protein folding simulations. Analysis of amyloid structures has to deal with the dynamics and flexibility of proteins, which must be capable of specific interactions with their intended ligands and substrates $[3,4]$. The emergence of amyloids is linked to the peculiarities of the folding process, which still awaits a comprehensive theoretical description-despite many decades of research [5].

In order to study such phenomena, we require techniques which would enable us to track the intermediate phases of folding. While traditional NMR is a useful tool, it requires soluble molecules [6], and that presents a problem when studying the pathogenicity of amyloids [7-9]. Much progress has recently been made owing to introduction of solid-state NMR [10]. One example is the elucidation of the structure of $A \beta(1-42)$, and particularly 
of its $A \beta(11-42)$ fragment, which is now listed in PDB [11]. This amyloid is the focus of the presented work. We subjected it to analysis from the point of view of hydrophobicity distribution. The presented work follows upon the results presented in [12], where we single out fragments exhibiting specific deviations from the theoretical ("idealized") distribution of hydrophobicity expected in a globular protein and mathematically defined by a $3 \mathrm{D}$ Gaussian [13-15].

The Gaussian distribution peaks at the center of an ellipsoid capsule. Its values decrease along with distance from the center, reaching nearly 0 on the surface. When the size of the capsule is adjusted to encapsulate the molecule in question, the corresponding Gaussian yields the theoretical (expected) values of hydrophobicity at any point within the protein body. This theoretical distribution is subsequently compared with the observed distribution of hydrophobicity, which depends on inter-residual interactions (themselves dependent on the mutual separation and intrinsic hydrophobicity of interacting residues). Differences between both distributions manifest themselves as either excess hydrophobicity or hydrophobicity deficiencies in specific areas of the protein body. The former-if present on the surface-mark protein complexation interfaces [16], while the latter are typically associated with ligand binding cavities [17] which enable the protein to perform its biological function [18].

To-date studies of amyloids point to a specific disagreement between the observed distribution of hydrophobicity and the theoretical (monocentric) Gaussian. In place of a central core, we are faced with linear propagation of repetitive patterns stretching along the fibril's main axis. Several fragments of the $A \beta(1-42)$ polypeptide have been identified as amyloid seeds - this includes the fragments at 11-16, 16-22 and 22-28 [12]. Consequently, our analysis will focus on these fragments in particular.

The $A \beta(11-42)$ sequence has been used as input for protein folding simulation toolkits, including I-Tasser [19-22] and Robetta [23, 24] — both highly ranked in the CASP competition $[25,26]$. Structures produced by both packages were assessed from the point of view of hydrophobicity distribution. In this respect, they proved to be highly diverse, ranging from near-globular to amyloid-like. In addition, we also generated several reference structures using software based on the fuzzy oil drop model (FOD), augmenting optimization of nonbonding interactions with alignment with an external (Gaussian) hydrophobic force field.

Each of the presented toolkits produced five structures, enabling us to compile a ranking list sorted by increasing differences between the observed structure and the theoretical Gaussian. This, in turn, shows how progressive deviations from the monocentric hydrophobic core model eventually cause the polypeptide chain to transform into a fibril, marked by alternating bands of high and low hydrophobicity. This analysis can be related to the reported polymorphism of amyloid structures [27].

This is why this paper can be treated as in silico experiment.

\section{Materials and methods}

\section{Protein under consideration}

Our analysis focused on the $A \beta(11-42)$ amyloid listed in PDB under ID 2MXU [11]. It represents the A $\beta(1-42)$ protein devoid of its $\mathrm{N}$-terminal fragment. The PDB structure comprises 12 separate polypeptides arranged into a fibril. This structural form is treated as reference one for all models delivered by mentioned programs. The status of complete fibrils, status of the chain as part of the fibril and one selected chain treated as individual structural unit are taken as the reference objects for those generated in silico.

\section{Folding of $A \beta(11-42)$}

The $A \beta(11-42)$ polypeptide was subjected to in silico folding simulations using two state-of-the-art protein folding toolkits: I-Tasser [19-22] and Robetta [23, 24]. Both packages consistently obtain high marks in the CASP [25] challenge and have been singled out as the most reliable computational tools currently available for this purpose $[22,23]$. The diversity of the resulting structures has its roots in the reported polymorphism of amyloid forms [27].

In addition to the above, our study set was extended with five structures generated by a custom toolkit based on the fuzzy oil drop model, which acknowledges the influence of the aqueous solvent upon the chain in question. This influence is modeled as an external force field with a Gaussian distribution, promoting internalization of hydrophobic residues along with exposure of hydrophilic residues on the surface [13-15]. As the application requires a starting structure (referred to as the Early Stage intermediate) we used the $2 \mathrm{MXU}$ structure as its input.

All resulting folds (15 in total; five per applicationpursuant to CASP criteria), along with the structure available in PDB, were subject to analysis of the shape of their hydrophobic cores in two separate contexts: as a composite fibril and as an individual chain from the $2 \mathrm{MXU}$ file.

FOD model was applied for protein folding for selected targets available in CASP6 (2004) [24]. We submitted models for 23 targets with the highest DTT_TS value equal to 41.98 . 


\section{Fuzzy oil drop model (FOD)}

The status of the hydrophobic core is expressed using the $\mathrm{RD}$ value which bases on the fuzzy oil drop model. This coefficient is calculated separately for two distinct variants: $\mathrm{RD}(\mathrm{T}-\mathrm{O}-\mathrm{R})$, which compares the observed distribution $(\mathrm{O})$ with two reference distributions: theoretical $(\mathrm{T})$ and uniform $(\mathrm{R})$, and $\mathrm{RD}(\mathrm{T}-\mathrm{O}-\mathrm{H})$, where the uniform distribution is replaced by a distribution which reflects the distribution expressed by intrinsic hydrophobicity of each residue. The definition of these parameters will be given below in the next part of Materials and Methods. In addition, three correlation coefficients of the above hydrophobicity distributions are computed: $\mathrm{H}$ versus $\mathrm{T}, \mathrm{T}$ versus $\mathrm{O}$ and $\mathrm{H}$ versus $\mathrm{O}$ called as $\mathrm{HvT}, \mathrm{TvO}$ and $\mathrm{HvO}$ in this paper.

Since a detailed presentation of the fuzzy oil drop model can be found in [13-15] we will limit ourselves to a brief recapitulation of its core concepts.

The input molecule is encapsulated in an ellipsoid used to calibrate the 3D Gaussian function, which, in turn, yields theoretical values of hydrophobicity at arbitrary points in the protein body. In contrast, the observed distribution depends on inter-residual interactions (as described in [28]). Both distributions ( $\mathrm{T}$ and $\mathrm{O}$ ) are measured at specific points which correspond to the so-called effective atoms (averaged-out positions of all atoms comprising each residue). In addition, each residue is assumed to represent certain intrinsic hydrophobicity as listed in [19], which is also used in observed hydrophobicity calculations.

In order to meaningfully compare alternative distributions, we apply the so-called Kullback-Leibler divergence entropy formula [29]. Since the result produced by this formula is a measure of entropy, it cannot be interpreted on its own-instead, it requires a reference value. This is why, in addition to $\mathrm{T}$, we introduce another reference distribution denoted $\mathrm{R}$, which stands from random. It is a uniform distribution which assigns every residue under consideration a hydrophobicity of $1 / \mathrm{N}$ where is $\mathrm{N}$ the number of these residues. Under these assumptions the RD value expresses the "closeness" of O to either T or R. Since-as discussed in [12] —in amyloid structures the observed distribution is dominated by the intrinsic properties of each participating residue, we also define another type of reference, denoted $\mathrm{H}$, which reflects the intrinsic hydrophobicity of each amino acid in the input chain. This results in two distinct values of $\mathrm{RD}$ : one for the T-O-R variant and one for the T-O-H variant. In the first case, a RD value greater or equal than 0.5 is taken as indication that protein's observed hydrophobicity profile does not follow the 3D Gaussian distribution, while in the other-that it explicitly follows the intrinsic model $(\mathrm{H})$.

As already mentioned, our comparative analysis also relies on three distinct correlation coefficients, providing a pairwise comparison of all distributions: $\mathrm{HvT}, \mathrm{TvO}$ and $\mathrm{HvO}$ (v stands from "versus"). Together, these coefficients express the influence of intrinsic hydrophobicity upon the structure of the hydrophobic core.

Globular proteins are usually closely aligned with $\mathrm{T}$ (this is particularly true for domains [30]), which means that hydrophobic residues are internalized while hydrophilic residues appear on the surface. Such conditions emerge as a result of "cooperation" between residues in an attempt to produce a common core. On the other hand, when residues act in a "selfish" manner, without cooperative tendencies, the result is a high value of $\mathrm{HvO}$ coupled with low (or even negative) values of both $\mathrm{TvO}$ and HvT. This suggests that the structure does not contain a monocentric core, and instead may exhibit other-in case of amyloid: linear propagation of repetitive patterns of hydrophobicity. Consequently, analysis of RD (together with the aforementioned correlation coefficients) may reveal progressive dilution of the hydrophobic core in favor of an entirely different structural pattern.

In order to further identify strongly amyloidogenic fragments, the above coefficients were also calculated for specific fragments of the input polypeptide.

Folding simulations were performed using computational resources provided by the Cyfronet $\mathrm{AGH}-\mathrm{Academic}$ Computing Center within the PL-Grid infrastructure. In fuzzy oil drop simulations the optimization of nonbonding interactions was carried out using the GROMACS package (also provided by Cyfronet) [31, 32].

\section{Results}

\section{Structure of the $A \beta(11-42)$ fibril}

The structure of the $A \beta(11-42)$ fibril may be assessed on the basis of $\mathrm{T}$ and $\mathrm{O}$ hydrophobicity distributions, as illustrated in Fig. 1. The figure reveals typical discordance between both distributions, with the observed hydrophobicity remaining high on the surface of the complex (contrary to expectations). In addition, a characteristic sinusoidal pattern is observed - the hallmark of a complex comprising multiple identical subunits arranged in a linear fashion, with alternating bands of high and low hydrophobicity exposed on the surface.

$\mathrm{RD}$ and correlation coefficients characterizing the fibrillary form will be discussed further on, in conjunction with the analysis of structures generated by in silico folding models.

The distribution of T shown in Fig. 1 reveals the characteristic concentration of hydrophobicity in the central part of fibril which is not followed by $\mathrm{O}$ distribution which represents sinusoid-like distribution along the whole fibril. The red lines do not represent the different $\mathrm{O}$ distributions. Only 
Fig. 1 Theoretical (T: blue) and observed (O: red) hydrophobicity distributions in the $\mathrm{A} \beta(11-42)$ fibril

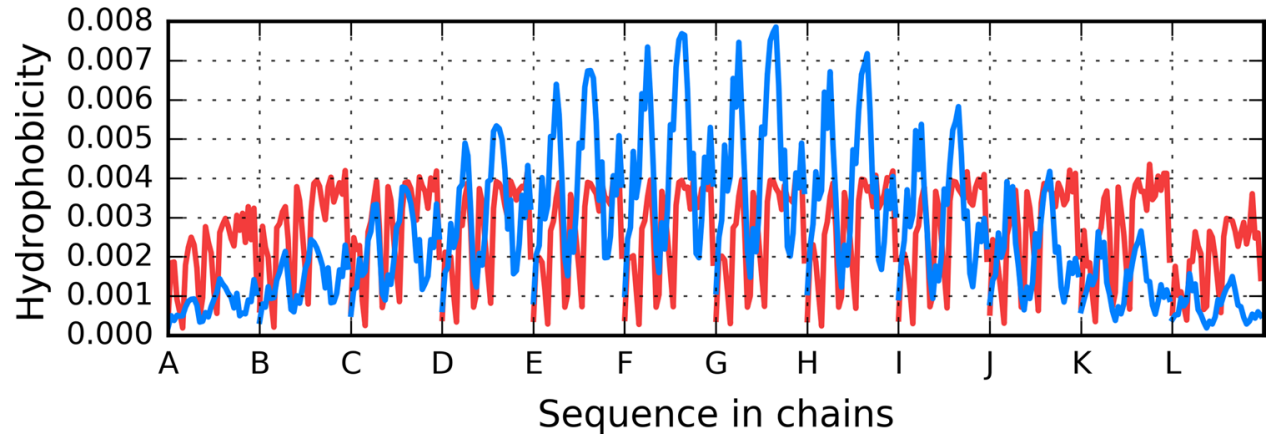

two $\mathrm{O}$ profiles can bees in Fig. 1a. This is due to overlapping of almost identical profiles for central polypeptides. Elimination of border polypeptide chains (chains A and L) visualizes it very clear (Fig. 2b). The T distributions for these polypeptides still represent different form depending of the position of polypeptide chain under consideration.

Since our analysis involves a complex which consists of a finite number of peptides, it does not accurately reflect the theoretical capability for unrestricted propagation. For this reason, we have singled out chain $\mathrm{F}$ (the central one) as representative of the fibril's structure. This chain was subsequently analyzed from two perspectives: as a part of the fibril (with 3D Gaussian fitted to the whole complex) and as an individual molecule (with 3D Gaussian fitted only to this chain and with disregard of other chains during $\mathrm{O}$ profile calculations). Figures $3 \mathrm{a}$ and $4 \mathrm{a}$ illustrate the theoretical, observed and intrinsic distributions for chain $\mathrm{F}$.
Besides sections where $\mathrm{T}$ is somewhat aligned with $\mathrm{O}$ (as well as with $\mathrm{H}$ ), there are areas where both distributions diverge notably. In order to identify these discordant sections, we calculated HvT, TvO and HvO correlation coefficients for individual 5 aa fragments using a moving frame approach, as shown in Fig. 3b and in Fig. 4b. These charts reveal fragments where all three coefficients adopt relatively high values. These fragments (residues 5 through 11) may be regarded as accordant with the 3D Gaussian model-they evidence the sort of cooperation which is required throughout the whole protein for a monocentric hydrophobic core to emerge.

The remaining fragments exhibit discordance which, in extreme cases, may produce a conformation which is a polar opposite of theoretical predictions (negative values of $\mathrm{TvO}$ and HvT). In these cases, the observed distribution is driven by the "selfish" tendencies of each residue rather
Fig. 2 Theoretical (T: blue) and observed (O: red) hydrophobicity distributions in the A $\beta(11-42)$ fibril presented in an overlapped mode (with all chains sharing the $\mathrm{X}$ axis): $\mathrm{A}$ all chains present in the fibril; $\mathbf{B}$ without one outlying chain from each end of the structure
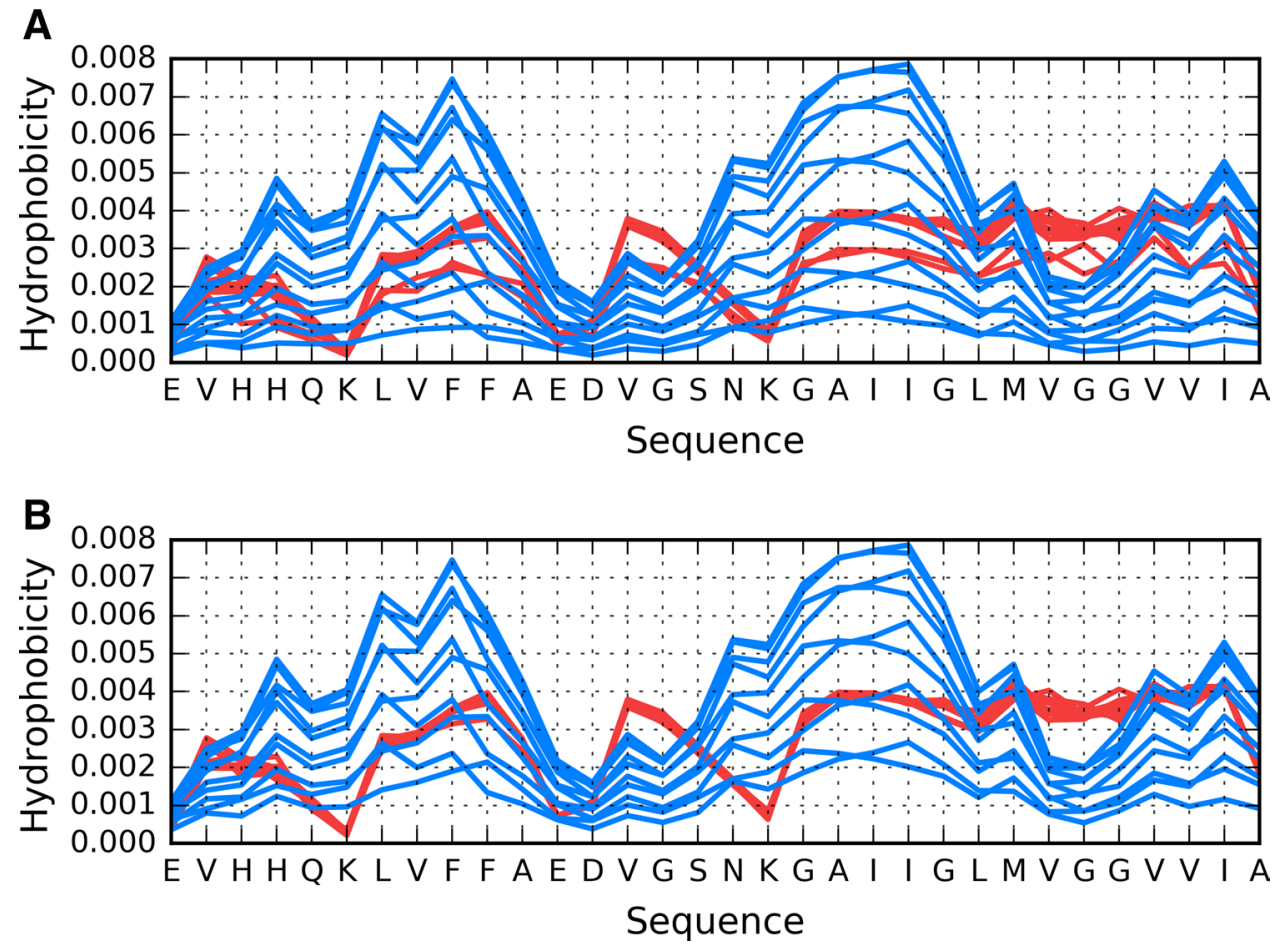
Fig. 3 Chain $\mathrm{F}$ analyzed as a component of the fibril: A T (blue), $\mathrm{O}$ (red) and $\mathrm{H}$ (green) hydrophobicity distributions; B correlation coefficients (HvO: blue, HvT: red, TvO: green) calculated for a 5 aa moving frame (in overlapped system). The indicated position on $\mathrm{X}$ axis represents the central residue in a given frame (i. e. 20 corresponds to residue 20 in the 18-19-20-21-22 frame)

Fig. 4 Chain $\mathrm{F}$ analyzed as an individual molecule: A T (blue), $\mathrm{O}$ (red) and $\mathrm{H}$ (green) hydrophobicity distributions; B correlation coefficients (HvO: blue, HvT: red, TvO: green) calculated for a 5 aa moving frame (in overlapped system). The indicated position on $\mathrm{X}$ axis represents the central residue in a given frame (i. e. 20 corresponds to residue 20 in the 18-19-20-21-22 frame)
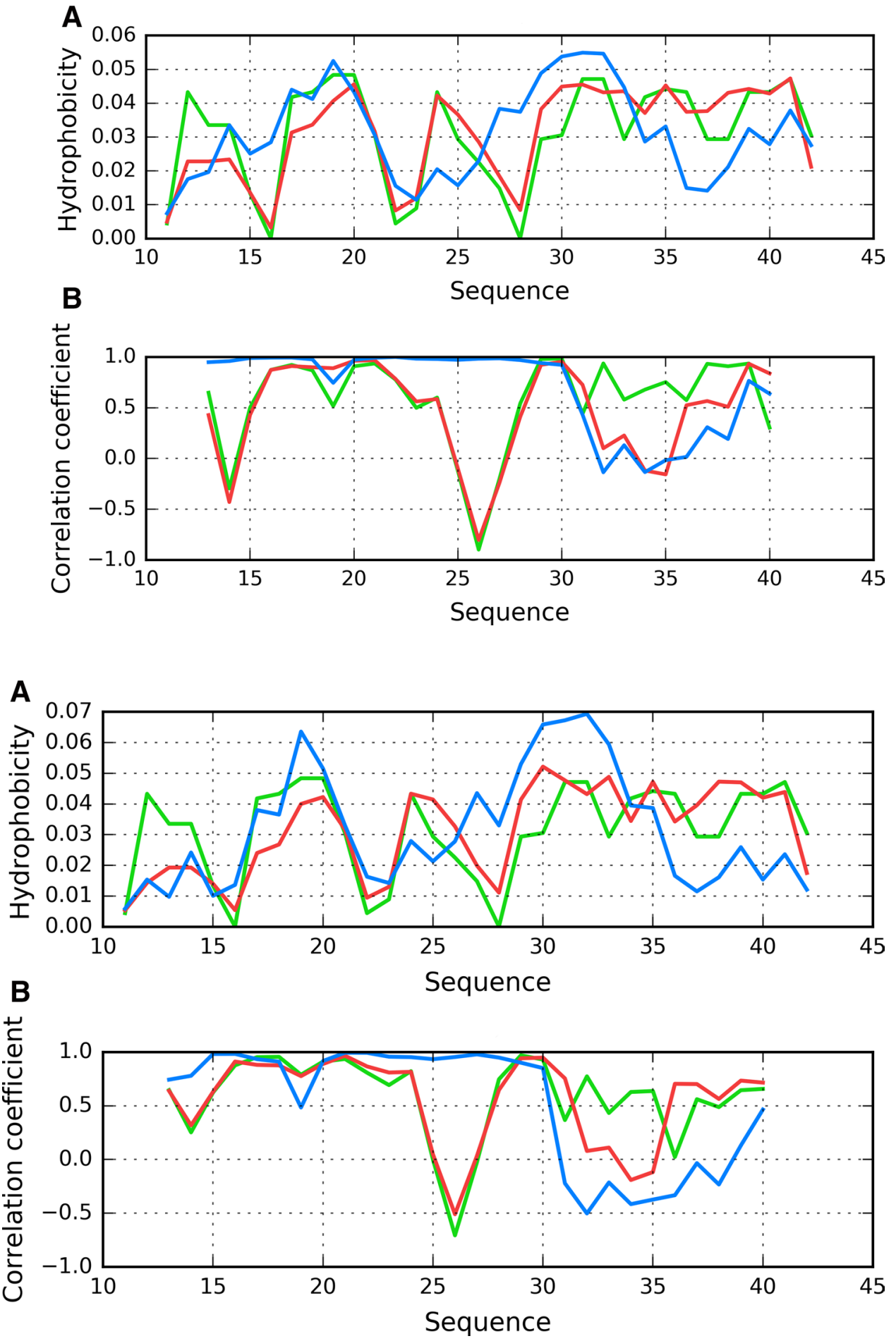

than by any tendency to produce a common hydrophobic core. Accordingly, we singled out fragments 11-16 and 24-28 as dominated by intrinsic hydrophobicity, while the 18-23 fragment may be described as locally accordant. Other fragments, while not exhibiting a clear preference for either $\mathrm{T}$ or $\mathrm{H}$, are included in our analysis to enable comparisons with structures produced by in silico tools. Their variable status (from globular to amyloid-like) will be discussed on the basis of RD and correlation coefficients.

Profiles observed for both views of chain F similarly reveal the presence of unexpected local maximum in the localization of expected local minimum in fragment 22-28. The discordant position of Lys 16 and particularly Lys 28 destroy the expected maximum introducing local minimum. Taking into account that this characteristics is continued 
along the whole fibril. This is why the positions of lysines play the contradictory role in respect to centralization of hydrophobicity. Calculation for both forms reveals the unusual distribution which can be even treated as contradictory status in respect to usual characteristics observed in globular proteins.

\section{Comparative analysis of protein structures generated by in silico folding models}

Structures generated by I-Tasser are labeled "I", those generated by Robetta are labeled "R", while those produced by the fuzzy oil drop software are labeled "F". All structures are numbered, with their respective numbers affixed to the source label. Results are listed in order of increasing values of $\mathrm{RD}(\mathrm{T}-\mathrm{O}-\mathrm{R})$ for the whole molecule, meaning that $\mathrm{F} 1$ is the FOD output with lowest value, R1-Robetta's, and so on.

Table 1 shows the full range of RD and correlation coefficients calculated for the entire molecule and for selected fragments. The list also includes chain F seen as a part of the fibril (labeled Af-amyloid/fibril, to avoid confusion with FOD results) and as an individual molecule (Ai-amyloid/ individual). Table 1 also shows the status of the 29-42 fragment, even though — while somewhat disordered—it does not resemble an amyloid seed [12].

According to Fig. 5, in models F1-R2, status of the 11-16 fragment is consistent with the 3D Gaussian distribution. This property, typical for globular proteins, fails to hold for model I1. In contrast, models R5 and I5 exhibit clear amyloid-like characteristics. Their HvO coefficients reach very high values, while the remaining coefficients are negative, indicating strong influence of intrinsic hydrophobicity. The set includes chain $\mathrm{F}$ analyzed as part of the complex (Af). Notably, under FOD classification criteria, model I4 exhibits the strongest amyloid affinity of the 11-16 fragment from among all analyzed structures. Its hydrophobicity profiles and 3D representation are shown on Fig. 6.

The summary shown in Fig. 7 shows that the 16-22 fragment in structure I3 exhibits amyloid-like characteristics, while structures F1-R4 are generally consistent with the theoretical distribution.

Structures shown in Fig. 8 visualize the hypothesis that the folding following intrinsical hydrophobicity directs the process toward the amlyoid-like structural forms. Two structural forms compared in Fig. 8 show that the 16-22 fragment may adopt a helical conformation, yet in an amyloid it becomes beta-like. In addition to that, these two models prove that the $A \beta(11-42)$ sequence may, in fact, produce a globule which can be seen to break apart as it transformation into an uncoiled loop (Fig. 8b) on its way to the amyloid form.
As illustrated in Fig. 9, the fragment at 24-28 becomes amyloid-like in structures Af (chain F in fibril) and I4. Analysis of distribution charts in this figure shows that in models F1, R1, R2 and R3 the fragment retains globular characteristics, while in model R4 it also resembles an amyloid. Sample 3D structures are visualized in Fig. 10.

From among the analyzed structures, F1's chain exhibits a particularly low $\mathrm{RD}(\mathrm{T}-\mathrm{O}-\mathrm{R})$ value, as shown in Fig. 11, where globular form was received with very little discordance between $\mathrm{T}$ and $\mathrm{O}$ profiles. In contrast, the highest $\mathrm{RD}(\mathrm{T}-\mathrm{O}-\mathrm{R})$ value was recorded for model I5's chain, with its $\mathrm{T}$ and $\mathrm{O}$ profiles illustrated in Fig. 12. To allow further analysis and comparison with other models, a selection of them is shown in Figs. 13 and 14. They visualize what kind of wide variety of structures can be produced by highly specialized programs.

In summary, we can single out structure I5 as potentially susceptible to linear propagation via complexation of identically folded polypeptides. Structure I5 (random coil structural form) also exhibits a propensity for complexation, however its distribution of hydrophobicity does not reveal the characteristic sinusoidal pattern observed in amyloids [12].

One can conclude that the main candidate for amyloid transformation in the $\mathrm{A} \beta(11-42)$ are two fragments: $11-16$ and 24-28. It can be seen in Table 1: the underlined sets of parameters denote models close to amyloid form: I4 and R4. These positions do not satisfy the condition of high value of $\mathrm{RD}(\mathrm{T}-\mathrm{O}-\mathrm{H})$. High value of this parameter requires a multichain complex as it is rather unusual for an isolated chain. This conclusion is correct on the condition of fuzzy oil drop acceptance as the method to trace the hydrophobic core identification and its transformation. This is why conclusion can be limited just to such a case.

\section{Discussion}

Summing up the presented results, we can state that the A $\beta(11-42)$ polypeptide may theoretically adopt various structural forms, including tightly packed globules characterized by high solubility (good agreement between $\mathrm{O}$ and $\mathrm{T}$ distributions). The set of candidate structures produced by I-Tasser and Robetta is highly diverse-from coherent globules all the way to disordered folds. Of particular note are the structured generated using the FOD model, where the presence of an external force field (aqueous solvent) drives the folding process towards the generation of a monocentric hydrophobic core-even though, under certain (so far unknown) conditions, this model may also produce strongly discordant structures.

Some of the obtained structures, e.g. I5 with RD(T-O$\mathrm{R})=0.768$, appears capable of forming complexes with other identically folded chains. The structure in question includes 
Table 1 Hydrophobicity-based parameters characterizing the structure of chain $\mathrm{F}$ from $2 \mathrm{MXU}$ in following forms: Af-when analyzed as a part of the fibril, Ai-when analyzed as an individual molecule,
I\#- as a result of simulation with I-Tasser, R\#-as a result of simulation with Robetta, F\#—as a result of simulation with FOD model

\begin{tabular}{|c|c|c|c|c|c|c|c|c|c|c|c|c|c|}
\hline \multirow[t]{2}{*}{ FORM } & \multicolumn{2}{|l|}{$\begin{array}{l}\text { RD } \\
11-42\end{array}$} & \multicolumn{3}{|l|}{$\begin{array}{l}\text { CC } \\
11-42\end{array}$} & \multicolumn{2}{|l|}{$\begin{array}{l}\text { RD } \\
11-16\end{array}$} & \multicolumn{4}{|c|}{$\begin{array}{l}\mathrm{CC} \\
11-16\end{array}$} & \multicolumn{2}{|l|}{$\begin{array}{l}\text { RD } \\
17-23\end{array}$} \\
\hline & T-O-R & T-O-H & HvT & $\mathrm{TvO}$ & $\mathrm{HvO}$ & T-O-R & T-O-H & & IvT & $\mathrm{TvO}$ & $\mathrm{HvO}$ & T-O-R & $\mathrm{T}-\mathrm{O}-\mathrm{H}$ \\
\hline $\mathrm{F} 1$ & 0.234 & 0.175 & 0.440 & 0.850 & 0.531 & 0.335 & 0.059 & & 0.188 & 0.653 & 0.807 & 0.138 & 0.119 \\
\hline $\mathrm{F} 2$ & 0.240 & 0.181 & 0.252 & 0.866 & 0.370 & 0.184 & 0.081 & & 0.309 & 0.961 & 0.486 & 0.194 & 0.155 \\
\hline F3 & 0.242 & 0.156 & 0.139 & 0.854 & 0.415 & 0.249 & 0.050 & & 0.380 & 0.835 & 0.773 & 0.169 & 0.083 \\
\hline $\mathrm{R} 1$ & 0.256 & 0.260 & 0.589 & 0.776 & 0.626 & 0.275 & 0.115 & & 0.807 & 0.828 & 0.977 & 0.330 & 0.326 \\
\hline $\mathrm{R} 2$ & 0.283 & 0.260 & 0.659 & 0.777 & 0.676 & 0.182 & 0.137 & & 0.751 & 0.941 & 0.810 & 0.354 & 0.329 \\
\hline $\mathrm{R} 3$ & 0.320 & 0.280 & 0.447 & 0.746 & 0.716 & 0.417 & 0.123 & & 0.376 & 0.485 & 0.953 & 0.387 & 0.384 \\
\hline I1 & 0.376 & 0.240 & 0.258 & 0.696 & 0.687 & 0.346 & 0.035 & & -0.208 & 0.717 & 0.257 & 0.304 & 0.248 \\
\hline $\mathrm{I} 2$ & 0.428 & 0.287 & 0.272 & 0.612 & 0.709 & 0.757 & 0.172 & & -0.512 & -0.111 & -0.597 & 0.473 & 0.348 \\
\hline R4 & 0.457 & 0.371 & 0.166 & 0.477 & 0.651 & 0.385 & 0.167 & & 0.226 & 0.628 & 0.871 & 0.187 & 0.191 \\
\hline I3 & 0.487 & 0.341 & 0.167 & 0.468 & 0.640 & 0.707 & 0.189 & & -0.481 & 0.015 & 0.531 & 0.757 & 0.509 \\
\hline $\mathbf{A i}$ & 0.536 & 0.519 & 0.408 & 0.567 & 0.698 & 0.488 & 0.220 & & 0.494 & 0.500 & 0.831 & 0.121 & 0.230 \\
\hline $\mathrm{F} 4$ & 0.555 & 0.285 & -0.073 & 0.412 & 0.329 & 0.534 & 0.064 & & -0.533 & 0.566 & 0.084 & 0.447 & 0.197 \\
\hline F5 & 0.559 & 0.226 & 0.154 & 0.391 & 0.260 & 0.533 & 0.054 & & -0.005 & 0.190 & 0.606 & 0.874 & 0.275 \\
\hline R5 & 0.660 & 0.521 & 0.172 & 0.254 & 0.641 & 0.655 & 0.201 & & 0.474 & -0.075 & 0.739 & 0.675 & 0.458 \\
\hline Af & 0.680 & 0.756 & 0.246 & 0.363 & 0.821 & 0.506 & 0.562 & & 0.121 & 0.257 & 0.966 & 0.133 & 0.473 \\
\hline I4 & 0.715 & 0.392 & -0.262 & 0.415 & 0.311 & 0.903 & 0.176 & & 0.469 & -0.452 & 0.788 & 0.464 & 0.166 \\
\hline I5 & 0.768 & 0.610 & -0.138 & 0.014 & 0.558 & 0.822 & 0.170 & & 0.259 & -0.118 & 0.680 & 0.826 & 0.720 \\
\hline \multirow[t]{2}{*}{ FORM } & \multicolumn{3}{|l|}{$\begin{array}{l}\mathrm{CC} \\
17-23\end{array}$} & \multicolumn{2}{|l|}{$\begin{array}{l}\text { RD } \\
24-28\end{array}$} & \multicolumn{3}{|l|}{$\begin{array}{l}\mathrm{CC} \\
24-28\end{array}$} & \multicolumn{2}{|l|}{$\begin{array}{l}\text { RD } \\
29-42\end{array}$} & \multicolumn{3}{|l|}{$\begin{array}{l}\mathrm{CC} \\
29-42\end{array}$} \\
\hline & HvT & $\mathrm{TvO}$ & $\mathrm{HvO}$ & T-O-R & T-O-H & HvT & $\mathrm{TvO}$ & $\mathrm{HvO}$ & T-O-R & T-O-H & HvT & $\mathrm{TvO}$ & $\mathrm{HvO}$ \\
\hline F1 & 0.632 & 0.939 & 0.716 & 0.269 & 0.251 & 0.845 & 0.866 & 0.965 & 0.264 & 0.189 & -0.030 & 0.824 & -0.307 \\
\hline $\mathrm{F} 2$ & 0.489 & 0.897 & 0.579 & 0.429 & 0.251 & -0.159 & 0.562 & 0.559 & 0.260 & 0.174 & -0.243 & 0.880 & -0.526 \\
\hline F3 & 0.092 & 0.908 & 0.440 & 0.353 & 0.179 & 0.521 & 0.717 & 0.912 & 0.168 & 0.123 & -0.386 & 0.910 & -0.306 \\
\hline $\mathrm{R} 1$ & 0.706 & 0.731 & 0.739 & 0.116 & 0.074 & 0.910 & 0.974 & 0.972 & 0.537 & 0.360 & -0.248 & 0.600 & -0.057 \\
\hline $\mathrm{R} 2$ & 0.608 & 0.653 & 0.863 & 0.367 & 0.091 & 0.566 & 0.718 & 0.937 & 0.433 & 0.431 & 0.675 & 0.650 & 0.373 \\
\hline R3 & 0.303 & 0.637 & 0.846 & 0.129 & 0.057 & 0.906 & 0.957 & 0.978 & 0.322 & 0.264 & 0.213 & 0.872 & 0.136 \\
\hline I1 & 0.416 & 0.808 & 0.826 & 0.530 & 0.092 & -0.167 & 0.243 & 0.854 & 0.618 & 0.675 & 0.259 & 0.674 & 0.514 \\
\hline I2 & 0.284 & 0.433 & 0.741 & 0.217 & 0.047 & 0.525 & 0.828 & 0.867 & 0.622 & 0.648 & 0.313 & 0.688 & 0.506 \\
\hline R4 & 0.766 & 0.959 & 0.632 & 0.705 & 0.192 & -0.641 & -0.633 & 0.978 & 0.575 & 0.486 & -0.199 & 0.536 & 0.085 \\
\hline I3 & -0.282 & -0.314 & 0.847 & 0.336 & 0.121 & 0.187 & 0.829 & 0.526 & 0.576 & 0.528 & 0.544 & 0.703 & 0.317 \\
\hline $\mathbf{A} \mathbf{i}$ & 0.907 & 0.925 & 0.888 & 0.669 & 0.382 & -0.509 & -0.706 & 0.954 & 0.864 & 0.789 & 0.100 & 0.504 & 0.110 \\
\hline F4 & 0.388 & 0.453 & 0.288 & 0.435 & 0.237 & -0.499 & 0.464 & 0.435 & 0.711 & 0.582 & -0.062 & 0.433 & -0.260 \\
\hline F5 & 0.155 & -0.762 & -0.047 & 0.606 & 0.135 & -0.633 & 0.019 & 0.628 & 0.536 & 0.322 & -0.337 & 0.566 & -0.739 \\
\hline R5 & 0.065 & -0.089 & 0.665 & 0.226 & 0.037 & 0.726 & 0.872 & 0.952 & 0.810 & 0.784 & 0.266 & 0.410 & 0.322 \\
\hline Af & 0.969 & 0.913 & 0.968 & 0.731 & 0.557 & -0.803 & -0.898 & 0.982 & 0.845 & 0.776 & 0.141 & 0.384 & 0.404 \\
\hline I4 & -0.879 & 0.444 & -0.194 & 0.702 & 0.133 & -0.787 & -0.840 & 0.949 & 0.765 & 0.660 & -0.045 & 0.705 & -0.104 \\
\hline I5 & -0.750 & -0.454 & 0.662 & 0.330 & 0.045 & 0.175 & 0.781 & 0.719 & 0.871 & 0.800 & 0.013 & -0.022 & -0.093 \\
\hline
\end{tabular}

Sequential numbers (1-5) after I, R and F sort models obtained with given program in order of increasing value of RD(T-O-R) calculated for the whole chain (residues 11-42). Parameters are given as as follows: RD for T-O-R and T-O-H variants, correlation coefficients (CC) for relations $\mathrm{H}$ versus $\mathrm{T}(\mathrm{HvT}), \mathrm{T}$ versus $\mathrm{O}(\mathrm{TvO})$ and $\mathrm{H}$ versus $\mathrm{O}(\mathrm{HvO})$. Id of structures given in bold distinguish fragment classified as amyloid; values italic are examples of forms close to amyloid form, understood as those expressing negative CC value of HvT and/or TvO with high CC value of $\mathrm{HvO}$ and with high $\mathrm{RD}$ values (in both variants) 
Fig. $5 \mathrm{HvO}$ (blue), HvT (red), $\mathrm{TvO}$ (green) calculated for residues 11-16 in successive structures as listed in Table 1
Fig. 6 Presentation of structure I4: A theoretical (T: blue) and observed (O: red) hydrophobicity profiles; B 3D view. Red highlight in $\mathbf{A}$ and red fragment in $\mathbf{B}$ correspond to 11-16 residue range
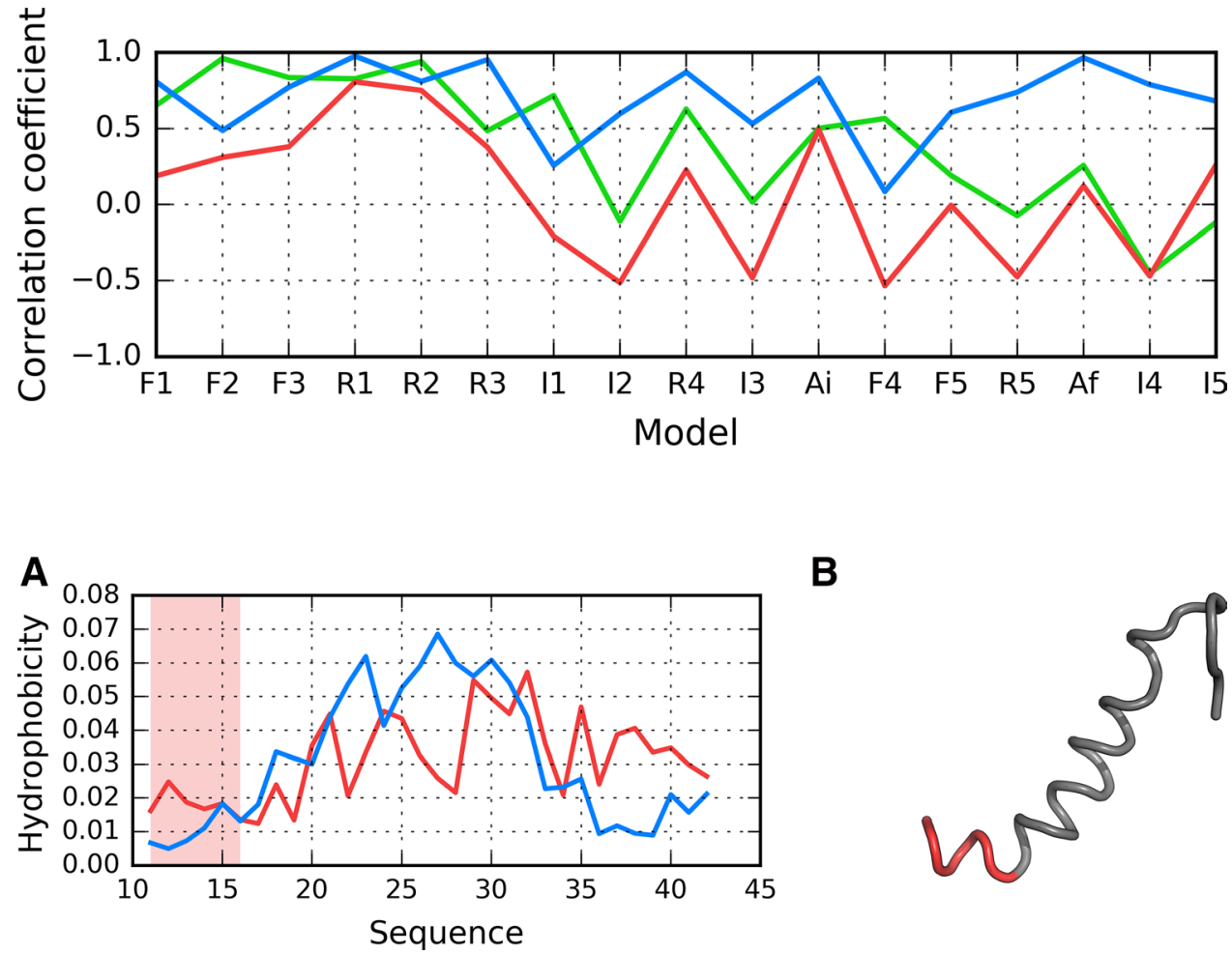

B
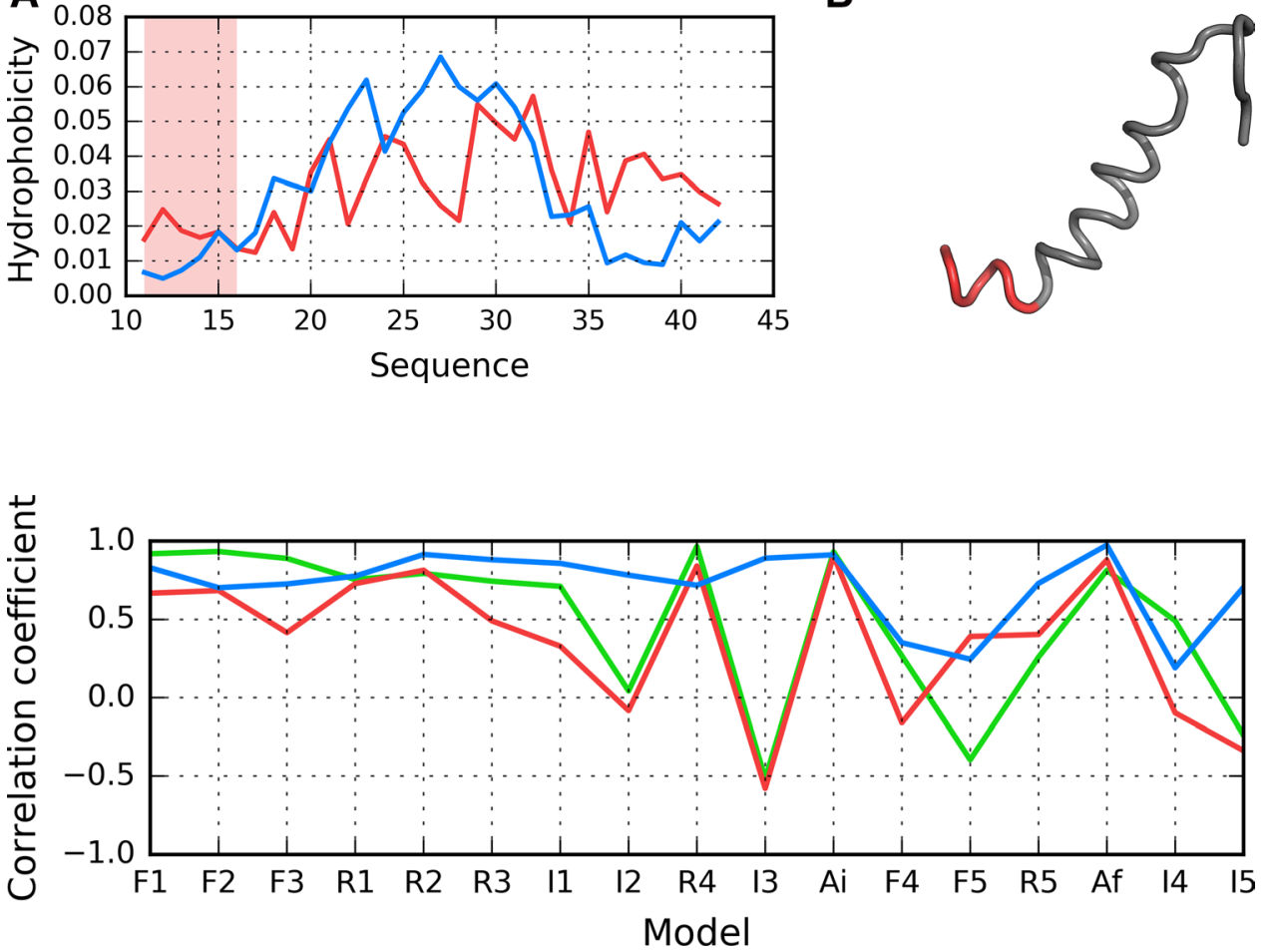

B

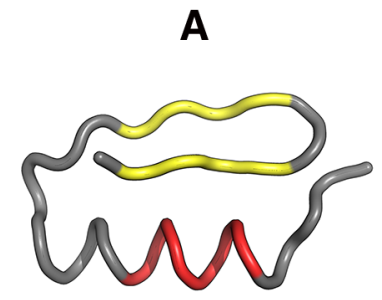

A

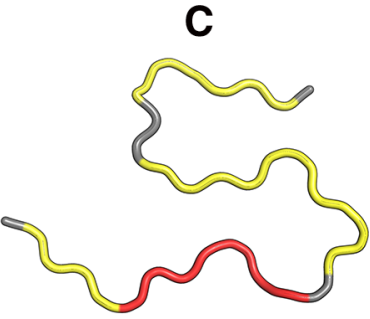

Fig. $7 \mathrm{HvO}$ (blue), HvT (red) TvO (green) calculated for residues 16-22 in successive structures as listed in Table 1
Fig. 8 3D view of structures R3 (A), I3 (B) and chain F (C) with 16-22 fragment colored red. Yellow fragments mark the locations of beta sheets (partially covered with red in $\mathbf{C}$ ) fragments which may be regarded as seeds of a conformational pattern guided by the intrinsic hydrophobicity of individual residues (negative correlation coefficients-see Table 1).

The interpretation provided in $[33,34]$ suggests that external conditions may support the misfolding not supporting the formation of centric hydrophobic core. The form of all hydrophobicity profiles as observed in amyloid forms analyzed here mark the positions of lysines as highly discordant in respect to what is expected by $\mathrm{T}$ distribution. The position of lysines introduces sharp local minimum in $\mathrm{O}$ in location where the local maximum is expected 
Fig. $9 \mathrm{HvO}$ (blue), HvT (red), $\mathrm{TvO}$ (green) calculated for residues 24-28 in successive structures as listed in Table 1
Fig. 10 3D view of structures R4 (A), I4 (B) and chain F (C) with 24-28 fragment colored red. Yellow fragments mark the locations of beta sheets

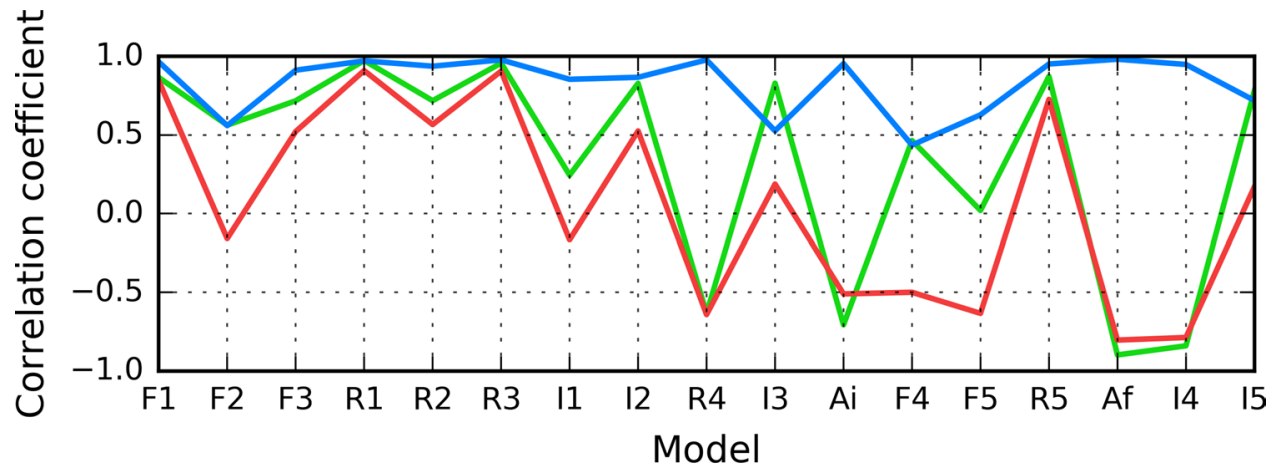

A

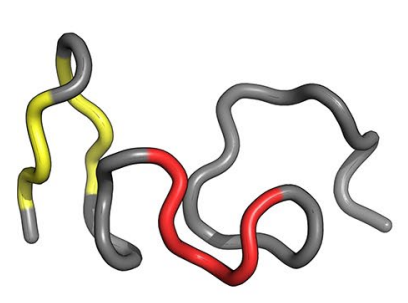

B

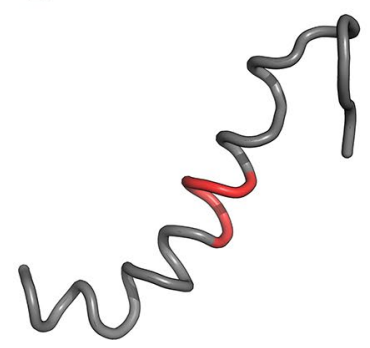

A

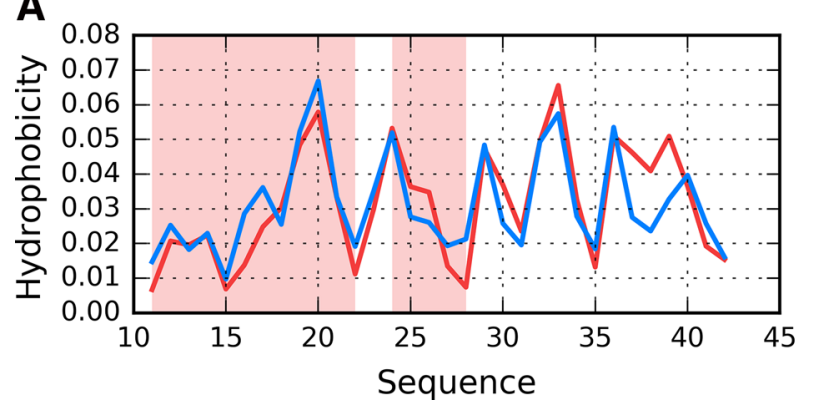

C

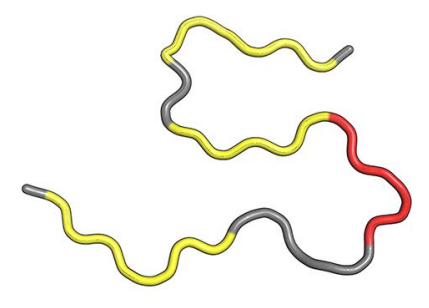

B

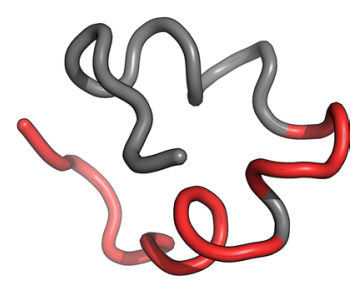

Fig. 11 Presentation of structure F1: A theoretical (T: blue) and observed (O: red) hydrophobicity profiles; B 3D view. Red highlights in $\mathbf{A}$ and red fragments in $\mathbf{B}$ correspond to 11-16, 16-22 and 24-28 residue ranges
Fig. 12 Presentation of structure I5: A theoretical (T: blue) and observed (O: red) hydrophobicity profiles; B 3D view. Red highlights in $\mathbf{A}$ and red fragments in $\mathbf{B}$ correspond to $11-16,16-22$ and $24-28$ residue ranges
A

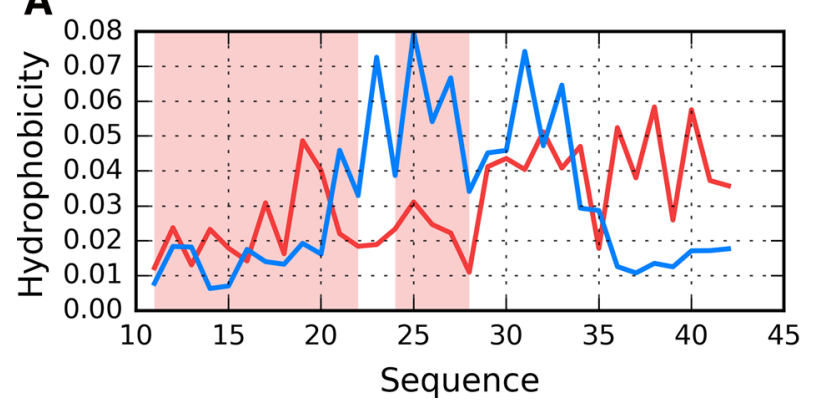

B

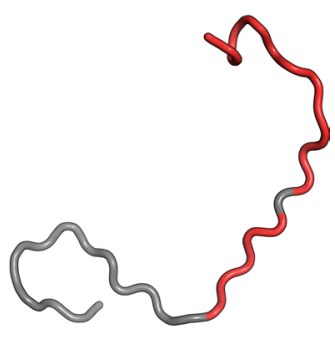

(due to the lowest intrinsic hydrophobicity in the scale). Especially position Lys28 introduces significant discordance. In this context, it is surprising to note the emergence of hydrophilic bands formed by linearly arranged lysine residues, as such conformations should be deterred through optimization of electrostatic interactions-and yet they can be observed under experimental conditions. In fragments characterized by negative correlation coefficients the central position is frequently occupied by lysine. When multiple fragments aggregate in a linear manner, 
Fig. 13 3D presentation of selected structures exhibiting $\mathrm{RD}(\mathrm{T}-\mathrm{O}-\mathrm{R})$ values calculated for the whole chain below 0.4 (exhibiting good accordance with FOD model): F2 (A), F3 (B) and I1 (C) with fragments 11-16, 16-22 and 24-28 in red

Fig. 14 3D presentation of structures accordant with the FOD model: R1 (A), R2 (B) contrasted with the amyloid structure-chain F (C) - with fragments 11-16, 16-22 and 24-28 in red. Yellow fragments mark the locations of beta sheets
A

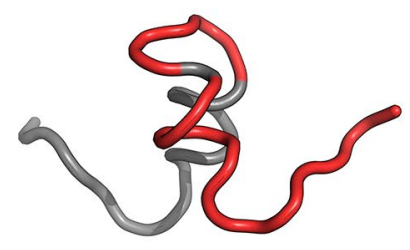

B

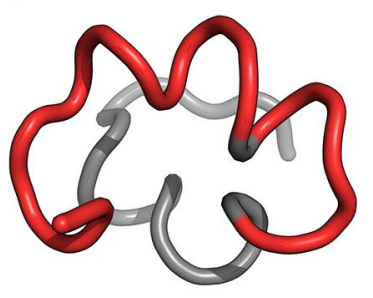

B
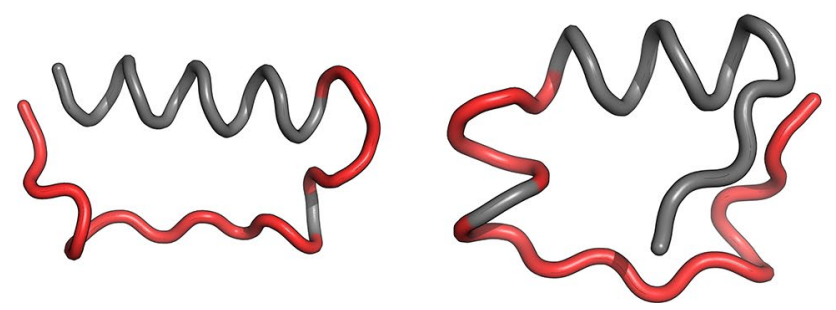

C

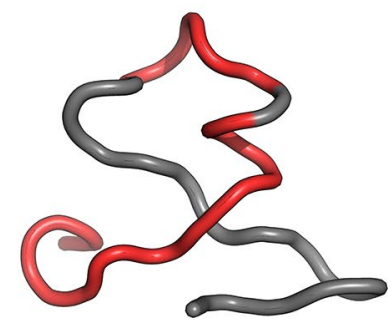

C

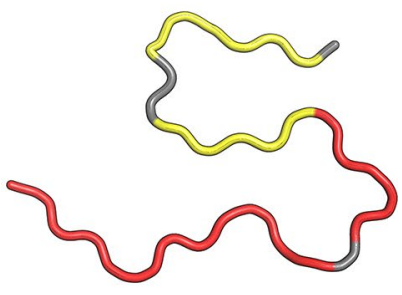

these lysines come into close contact with one another, forming a hydrophilic band. This shows that hydrophobic forces override electrostatic interactions, and that disruptions in the external force field may produce a conformation which depends on the intrinsic hydrophobicity of participating residues. The above interpretation provides indirect confirmation of the correctness of the fuzzy oil drop model, which, under ordinary circumstances, leads to the emergence of a monocentric hydrophobic core encapsulated by a hydrophilic "shell". The importance of hydrophobic interactions for seeding amyloid transformation has already been noted in $[35,36]$. In [36] the authors suggest that amyloid transformation may result from insufficient influence of the external force field (water) upon the folding process. This conclusion is consistent with the interpretation of results obtained using the fuzzy oil drop model, as presented in this paper.

In conclusion based on the presented model the weakening of standard external force field (water environment) prevents it from driving the forlding process toward centralization of hydrophobic residues and allows the intrinsic hydrophobicity to dominate.

The effect in this case is the micellarization. In particular, a ribbon-like micelle is preferred despite non-favorable interaction of charged residues arranged in electrostatically suboptimal form. The arguments for this conclusion are coming from in vitro experiments-especially shaking, which introduces air to water and-in consequence-much higher presence of inter-phase-related order of water molecules. This is why the structure of water in its standard form as well as under influence of external factors (not necessarily of chemical character) should be in focus of research oriented on amyloid transformation.

Acknowledgements The authors are indebted to Piotr Nowakowski for translation services, and to Anna Śmietańska for editorial work. This work was supported by the Jagiellonian University Collegium Medicum Grant No. K/ZDS/006363.

Author contributions I.R., and L.K.: Conceptualization; D.D., and M.G.: Methodology; M.B., and M.G.: Software; D.D., and M.G.: Validation; I.R.: Formal Analysis; I.R.: Investigation; I.R., and M.B.: Resources; I.R.: Writing-Original Draft Preparation; I.R., and M.B.: Writing-Review \& Editing; M.B.: Visualization; L.K.: Supervision.

Open Access This article is distributed under the terms of the Creative Commons Attribution 4.0 International License (http://creativeco mmons.org/licenses/by/4.0/), which permits unrestricted use, distribution, and reproduction in any medium, provided you give appropriate credit to the original author(s) and the source, provide a link to the Creative Commons license, and indicate if changes were made.

\section{References}

1. Chiti F, Dobson CM (2017) Protein misfolding, amyloid formation, and human disease: a summary of progress over the last decade. Annu Rev Biochem 86(1):27-68. https://doi.org/10.1146/ annurev-biochem-061516-045115

2. Eisenberg DS, Sawaya MR (2017) Structural studies of amyloid proteins at the molecular level. Annu Rev Biochem 86(1):69-95. https://doi.org/10.1146/annurev-biochem-061516-045104

3. Joseph AP, de Brevern AG (2014) From local structure to a global framework: recognition of protein folds. J R Soc Interface 11(95):20131147. https://doi.org/10.1098/rsif.2013.1147

4. Stirnemann G, Giganti D, Fernandez JM, Berne BJ (2013) Elasticity, structure, and relaxation of extended proteins under force. Proc Natl Acad Sci U S A 110(10):3847-3852. https://doi.org/10.1073/ pnas. 1300596110

5. Khoury GA, Liwo A, Khatib F, Zhou H, Chopra G, Bacardit J, Bortot LO, Faccioli RA, Deng X, He Y, Krupa P, Li J, Mozolewska MA, Sieradzan AK, Smadbeck J, Wirecki T, Cooper S, 
Flatten J, Xu K, Baker D, Cheng J, Delbem AC, Floudas CA, Keasar C, Levitt M, Popović Z, Scheraga HA, Skolnick J, Crivelli SN, Foldit Players (2014) WeFold: a coopetition for protein structure prediction. Proteins 82(9):1850-1868. https://doi. org/10.1002/prot. 24538

6. Hu K-N, Tycko R (2010) What can solid state NMR contribute to our understanding of protein folding? Biophys Chem 151(12):10-21. https://doi.org/10.1016/j.bpc.2010.05.009

7. Tay WM, Huang D, Rosenberry TL, Paravastu AK (2013) The Alzheimer's amyloid- $\beta(1-42)$ peptide forms off-pathway oligomers and fibrils that are distinguished structurally by intermolecular organization. J Mol Biol 425(14):2494-2508. https://doi. org/10.1016/j.jmb.2013.04.003

8. Rasmussen J, Mahler J, Beschorner N, Kaeser SA, Häsler LM, Baumann F, Nyström S, Portelius E, Blennow K, Lashley T, Fox NC, Sepulveda-Falla D, Glatzel M, Oblak AL, Ghetti B, Nilsson KPR, Hammarström P, Staufenbiel M, Walker LC, Jucker M (2017) Amyloid polymorphisms constitute distinct clouds of conformational variants in different etiological subtypes of Alzheimer's disease. Proc Natl Acad Sci U S A 114(49):13018-13023. https://doi.org/10.1073/pnas.1713215114

9. Eisenberg D, Jucker M (2012) The amyloid state of proteins in human diseases. Cell 148(6):1188-1203. https://doi.org/10.1016/j. cell.2012.02.022

10. Tycko $\mathrm{R}$ (2016) Molecular structure of aggregated amyloid- $\beta$ : insights from solid-state nuclear magnetic resonance. Cold Spring Harb Perspect Med 6(8):a024083. https://doi.org/10.1101/cshpe rspect.a024083

11. Xiao Y, Ma B, McElheny D, Parthasarathy S, Long F, Hoshi M, Nussinov R, Ishii Y (2015) A beta (1-42) fibril structure illuminates self-recognition and replication of amyloid in Alzheimer's disease. Nat Struct Mol Biol 22(6):499-505. https://doi. org/10.1038/nsmb.2991

12. Dułak D, Banach M, Konieczny L, Roterman I (2018) Structural analysis of the $\mathrm{a} \beta(15-40)$ amyloid fibril based on hydrophobicity distribution. Acta Biochim Pol 65(4):595-604. https://doi. org/10.18388/abp.2018_2647

13. Konieczny L, Bryliński M, Roterman I (2006) Gauss-functionbased model of hydrophobicity density in proteins. Silico Biol 6(1-2):15-22

14. Kalinowska B, Banach M, Konieczny L, Roterman I (2015) Application of divergence entropy to characterize the structure of the hydrophobic core in DNA interacting proteins. Entropy 17(3):1477-1507. https://doi.org/10.3390/e17031477

15. Roterman I, Banach M, Konieczny L (2017) Application of the fuzzy oil drop model describes amyloid as a ribbonlike micelle. Entropy 19(4):167. https://doi.org/10.3390/e19040167

16. Banach M, Konieczny L, Roterman I. Use of the fuzzy oil drop model to identify the complexation area in protein homodimers. In: I. Roterman-Konieczna (Eds), Protein folding In silico, Woodhead Publishing (currently Elsevier), Oxford, Cambridge, Philadelphia, New Dehli, 2012, pp 95-122, https://doi. org/10.1533/9781908818256.95

17. Banach M, Konieczny L, Roterman I. Ligand-binding-site recognition. In: I. Roterman-Konieczna (Eds), Protein folding In silico, Woodhead Publishing (currently Elsevier), Oxford, Cambridge, Philadelphia, New Dehli, 2012, pp 79-93, https://doi. org/10.1533/9781908818256.79

18. Banach M, Konieczny L, Roterman I (2014) The fuzzy oil drop model, based on hydrophobicity density distribution, generalizes the influence of water environment on protein structure and function. J Theor Biol 359:6-17. https://doi.org/10.1016/j. jtbi.2014.05.007
19. Zhang Y (2008) I-TASSER server for protein 3D structure prediction. BMC Bioinformatics 9(1):40. https://doi. org/10.1186/1471-2105-9-40

20. Roy A, Kucukural A, Zhang Y (2010) I-TASSER: a unified platform for automated protein structure and function prediction. Nat Protoc 5(4):725-738. https://doi.org/10.1038/nprot.2010.5

21. Yang J, Yan R, Roy A, Xu D, Poisson J, Zhang Y (2015) The I-TASSER suite: protein structure and function prediction. Nat Methods 12(1):7-8. https://doi.org/10.1038/nmeth.3213

22. https://zhanglab.ccmb.med.umich.edu/I-TASSER. Accessed 20 July 2018

23. http://robetta.org. Accessed 20 July 2018

24. Kim DE, Chivian D, Baker D (2004) Protein structure prediction and analysis using the Robetta server. Nucleic Acids Res 32(Web Server):W526-W531. https://doi.org/10.1093/nar/gkh468

25. http://predictioncenter.org. Accessed 20 July 2018

26. Moult J, Fidelis K, Kryshtafovych A, Schwede T, Tramontano A (2013) Critical assessment of methods of protein structure prediction (CASP)—round x. Proteins 82:1-6. https://doi.org/10.1002/ prot.24452

27. Qiang W, Kelley K, Tycko R (2013) Polymorph-specific kinetics and thermodynamics of $\beta$-amyloid fibril growth. J Am Chem Soc 135(18):6860-6871. https://doi.org/10.1021/ja311963f

28. Levitt MA (1976) A simplified representation of protein conformations for rapid simulation of protein folding. J Mol Biol 104(1):59-107. https://doi.org/10.1016/0022-2836(76)90004-8

29. Kullback S, Leibler RA (1951) On information and sufficiency. Ann Math Stat 22(1):79-86. https://doi.org/10.1214/aoms/11777 29694

30. Sałapa K, Kalinowska B, Jadczyk T, Roterman I (2012) Bio-algorithms and med-systems measurement of hydrophobicity distribution in proteins-non-redundant protein data Bank. BAMS. https ://doi.org/10.2478/bams-2012-0023

31. Berendsen HJC, van der Spoel D, van Drunen R (1995) GROMACS: a message-passing parallel molecular dynamics implementation. Comput Phys Commun 91(1-3):43-56. https:// doi.org/10.1016/0010-4655(95)00042-e

32. http://www.gromacs.org. Accessed 20 July 2018

33. Roterman I, Banach M, Kalinowska B, Konieczny L (2016) Influence of the aqueous environment on protein structure-a plausible hypothesis concerning the mechanism of amyloidogenesis. Entropy 18(10):351. https://doi.org/10.3390/e18100351

34. Das AK, Rawat A, Bhowmik D, Pandit R, Huster D, Maiti S (2015) An early folding contact between Phe19 and Leu34 is critical for amyloid- $\beta$ oligomer toxicity. ACS Chem Neurosci 6(8):1290-1295. https://doi.org/10.1021/acschemneuro.5b00074

35. Paravastu AK, Qahwash I, Leapman RD, Meredith SC, Tycko R (2009) Seeded growth of beta-amyloid fibrils from Alzheimer's brain-derived fibrils produces a distinct fibril structure. Proc Natl Acad Sci U S A 106(18):7443-7448. https://doi.org/10.1073/ pnas.0812033106

36. Schmit JD, Ghosh K, Dill K (2011) What drives amyloid molecules to assemble into oligomers and fibrils? Biophys $\mathrm{J}$ 100(2):450-458. https://doi.org/10.1016/j.bpj.2010.11.041

Publisher's Note Springer Nature remains neutral with regard to jurisdictional claims in published maps and institutional affiliations. 